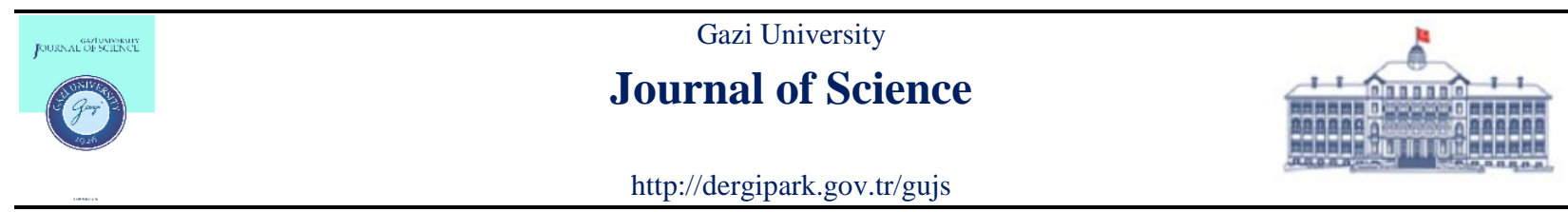

\title{
A New Family of Archimedean Copula via Trigonometric Generator Function
}

\author{
Selim Orhun SUSAM* \\ Munzur University, Faculty of Economics and Administrative Sciences, Department of Econometrics, 62200, Tunceli, Turkey
}

\section{Highlights}

- A new type of Archimedean copula is proposed.

- Proposed copula covers wider dependence coefficients interval.

- We investigated the estimation of the parameter of cotangent copula.

- Cotangent copula can lead to better fits for real data.

\section{Article Info}

Received: 20/10/2019

Accepted: 03/04/2020

\section{Keywords}

Archimedean copula

Tail dependence

Kendall's tau

Parameter estimation

\begin{abstract}
In this paper, new type of Archimedean copula based on cotangent generator function, and dependence characteristics are investigated. Also, the estimation procedure of the dependence parameter is given. A Monte Carlo study is performed to measure the performance of estimators. To demonstrate the goodness of fit performance of the new family, a real data analysis is performed.
\end{abstract}

\section{INTRODUCTION}

Copula models are popular tools for describing the dependence of multivariate data where the univariate distribution functions are combined with joint distribution function by Sklar's theorem (Sklar [1]). Consider random variables $\boldsymbol{X}$ and $\boldsymbol{Y}$ with joint cumulative distribution function $\boldsymbol{H}$ and marginals $\boldsymbol{F}$ and $\boldsymbol{G}$, respectively. Then there exists a copula $\mathrm{C}$ such that $\boldsymbol{H}(\boldsymbol{x}, \boldsymbol{y})=\boldsymbol{C}(\boldsymbol{F}(\boldsymbol{x}), \boldsymbol{G}(\boldsymbol{y}))$, for all $\boldsymbol{x}, \boldsymbol{y}$.

If the margins are continuous, then $C$ is unique; otherwise, $C$ is uniquely determined on $\operatorname{Range}(F) \times$ Range $(G)$. When the bivariate joint distribution function $H$ and the marginals $F$ and $G$ are known, copula can be constructed as $C(u, v)=H\left(F^{-1}(u), G^{-1}(v)\right)$ where $U=F(x)$ and $V=G(y)$.

A bivariate copula $C:[0,1]^{2} \rightarrow[0,1]$ is a bivariate distribution function with the following properties:

- $C(u, 0)=C(0, v)=0$,

- $\quad C(u, 1)=u$ and $C(1, \mathrm{v})=\mathrm{v}$,

- $\mathrm{C}$ is two-increasing, that is, for every rectangle $B:\left[u_{1}, u_{2}\right] \times\left[v_{1}, v_{2}\right]$ in $I$ such that $u_{1} \leq u_{2}$ and $\mathrm{v}_{1} \leq \mathrm{v}_{2}$. The $\mathrm{C}$-Volume of $\mathrm{B}$ is non-negative, i.e.

$$
V_{C}(B)=C\left(u_{2}, v_{2}\right)-C\left(u_{2}, v_{1}\right)-C\left(u_{1}, v_{2}\right)+C\left(u_{1}, v_{1}\right) \geq 0 \text {. }
$$

In the copula literature, an important family of copulas is known as Archimedean copula. This family of copulas class is characterized by the generator functions $\varphi$. Archimedean copula with generator function $\varphi$, $C: I^{2} \rightarrow I$ is defined by

$C(u, v)=\varphi^{[-1]}\{\varphi(u)+\varphi(v)\} ; u, v \in[0,1]$. 
The generator function $\varphi$ has the following properties

1) $\varphi(1)=0$,

2) $\varphi$ is decreasing, that is $\varphi^{\prime}(t)<0, t \in(0,1)$,

3) $\varphi$ is strictly convex, that is $\varphi^{\prime \prime}(t)>0, t \in(0,1)$.

In recent years, various studies have been carried out on the Archimedean copula with trigonometric generator function. First time in the literature, Bal and Najjari [2] introduced Archimedean copula with hyperbolic cosecant generator function as $\varphi_{\operatorname{csch}}(t)=\operatorname{csch}\left(t^{\theta}\right)-\operatorname{csch}(1), \theta>0$. Najjari et al. [3] proposed hyperbolic cotangent function as a generator of new Archimedean copula as $\varphi_{\text {coth }}(t)=$ $\operatorname{coth}(t \theta)-\operatorname{coth}(\theta), \theta>0$. Some dependence properties of this new family were investigated in their work. In our opinion, there are some disadvantages to the usage of $\varphi_{c s c h}(t)$ and $\varphi_{c o t h}(t)$. As stated in Bal and Najjari [2], general dependence measure Kendall's tau cannot be obtained as a closed-form for $\varphi_{\text {csch }}(t)$. So, they derived approximated formula of Kendall's tau. Despite Najjari et al. [3] obtained a closed-form of Kendall's tau, interval of Kendall's tau covers [1/3,1]. On the other hand, lower tail dependence is constant and equals to $1 / 2$. In this study, a cotangent function is proposed as a new trigonometric generator function. This new trigonometric copula covers wider lower tail dependence and Kendall's tau interval than the hyperbolic cotangent copula. In addition, all dependence measures can be derived as a closed-form.

The rest of the paper is organized as follows. Section 2 gives some introduction on the existing Archimedean copula with trigonometric function and introduces a new family of Archimedean copula. Section 3 is devoted to the parameter estimation of the new family. Also, the Monte Carlo simulation study is performed to measure the performance of estimators. Section 3 describes the application to real data. Finally, the last section is devoted to the conclusion.

\section{COTANGENT GENERATOR FUNCTION AND ITS PROPERTIS}

Any generator function of the Archimedean copula must be satisfied to properties given in definition 1 . In summary, $\varphi(\mathrm{t})$ has some properties such as $\varphi(1)=0, \varphi^{\prime}(t)<0, \varphi^{\prime \prime}(t) \geq 0$. Let $\varphi_{\cot }(t)=\cot (t)^{\theta}-$ $\cot (1)^{\theta}$ be our proposed trigonometric generator function. Our new trigonometric generator function has two continuous derivatives on $(0,1)$, and it satisfies to all properties of generator function for all $\theta \in(0, \infty)$. The $\varphi_{\cot }(\mathrm{t})$ with different parameters displayed in Figure 1. Its inverse function can be defined as

$\varphi^{-1}(t)=\operatorname{arccot}\left(\left(\cot (1)^{\theta}+t\right)^{\frac{1}{\theta}}\right)$

so that, related copula can be defined as

$C(u, v)=\operatorname{arccot}\left(\left(\cot (u)^{\theta}+\cot (v)^{\theta}-\cot (1)^{\theta}\right)^{\frac{1}{\theta}}\right)$.

For dependence measures, Kendall's tau can be expressed as follows

$$
\tau=1+4 \int_{0}^{1} \int_{0}^{1} C(u, v) d C(u, v)=1+4 \int_{0}^{1} \frac{\varphi(t)}{\varphi^{\prime}(t)} d t=1-\frac{2_{1}^{2} F\left(1, \frac{2+\theta}{2}, \frac{4+\theta}{2},-\tan (1)^{2}\right)}{2+\theta} \tan (1)^{2}
$$




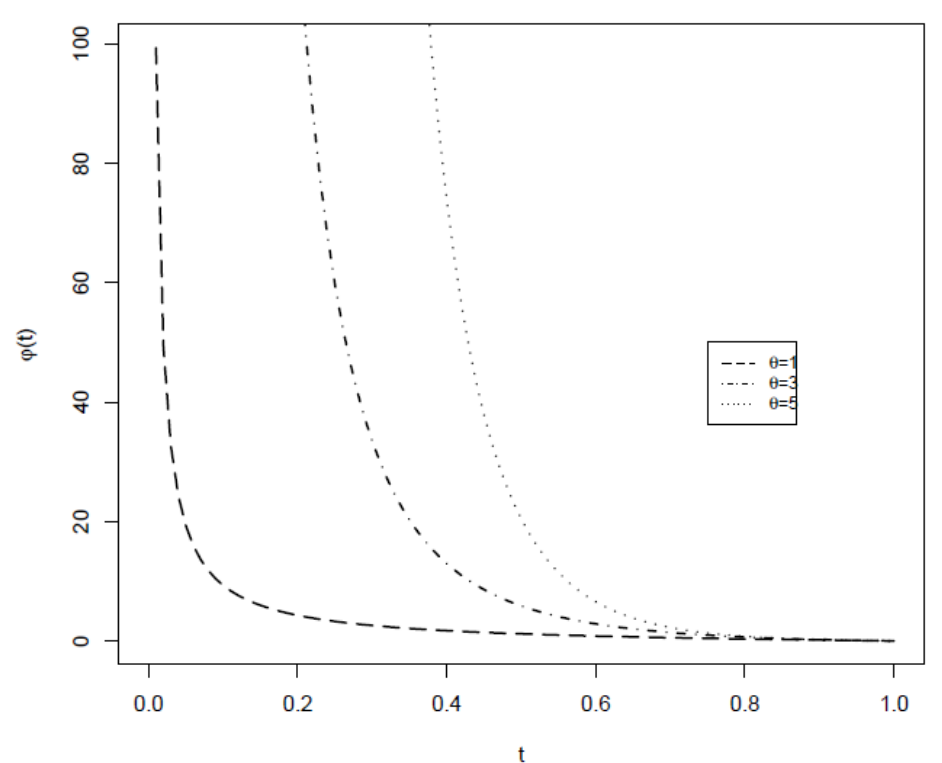

Figure 1. $\varphi_{\cot }(t)$ with $\theta=1 ; 3 ; 5$

where ${ }_{1}^{2} F$ is the hypergeometric function. For more details about the hypergeometric function, see Bagdasaryan [4]. All results are derived scientific computing program "Mathematica 11.0.1.0"'. Graph of the function $\tau(\theta)$ displayed in Figure 2(a). From Figure 2(a), $\lim _{\theta \rightarrow \infty} \tau(\theta)=1$. As for tail behavior, upper tail dependence and lower tail dependence coefficients can be expressed in terms of generator function, respectively, as

$\lambda_{U}=2-2^{\lim _{t \rightarrow 1}\left(\frac{\varphi(t)}{\varphi^{\prime}(t)}\right)^{\prime}}=0, \lambda_{L}=2^{\lim _{t \rightarrow 0^{+}}\left(\frac{\varphi(t)}{\varphi^{\prime}(t)}\right)^{\prime}}=2^{-\frac{1}{\theta}}$.

See Michiels et al. [5]. Graph of the function $\lambda_{L}(\theta)$ displayed in Figure 2(b). From Figure 2(b), $\lim _{\theta \rightarrow \infty} \lambda_{L}(\theta)=1$. Also, Figure 3 displays scatter plots of new trigonometric copula with dependence parameters $\theta=1 ; 3 ; 5$. We conclude that it behaves much like the Clayton family. Also, dependence coefficients range of trigonometric Archimedean copula is given in Table 1.

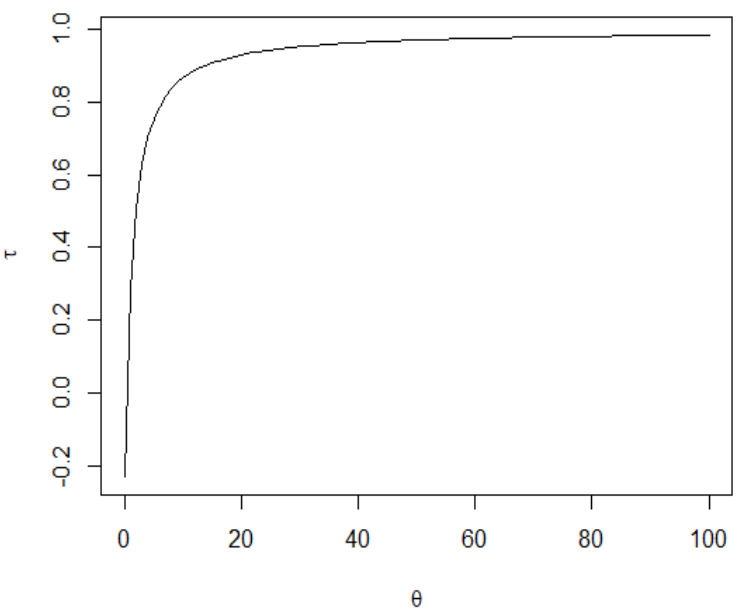

(a) Graphs of the function $\tau(\theta)$

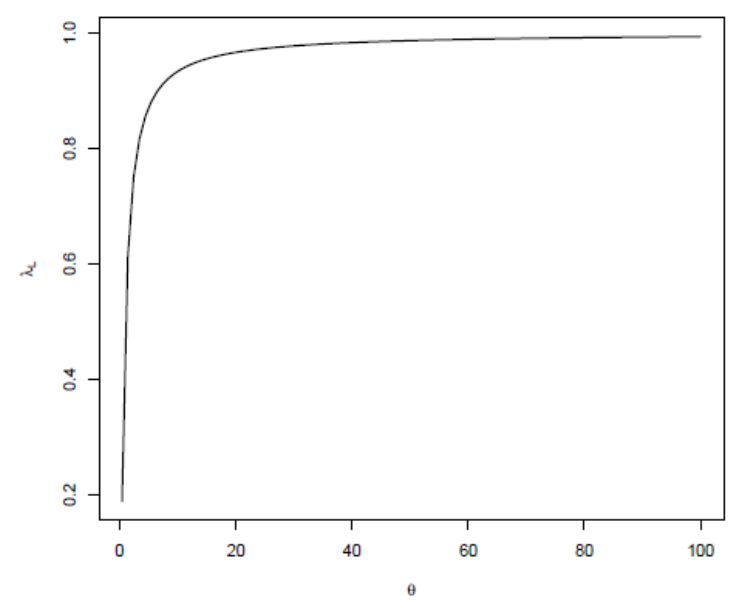

(b) Graphs of the function $\lambda_{L}(\theta)$

Figure 2. Graphs of the dependence coefficients 


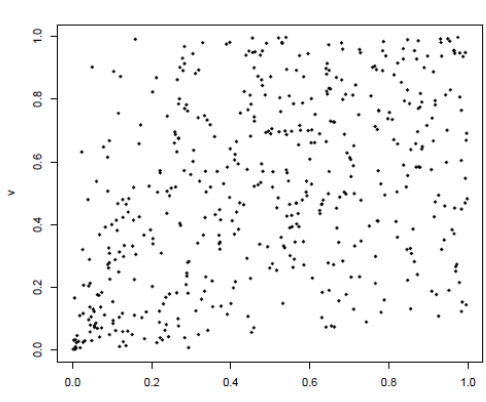

(a) $\theta=1$

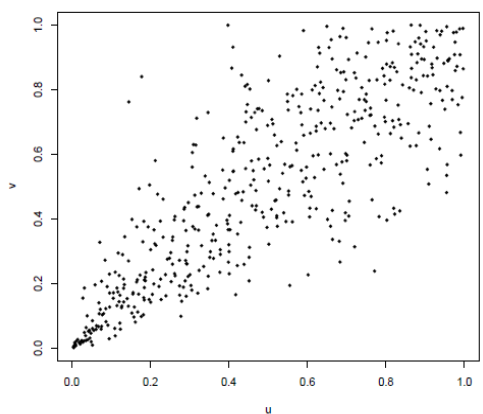

(b) $\theta=3$

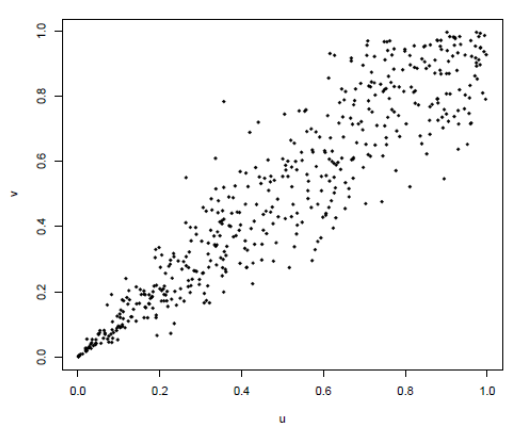

(c) $\theta=5$

Figure 3. Scatter plots of new family

When Table 1 examined, the new Cotangent copula covers a larger lower tail dependence interval than Cotangent hyperbolic copula. Also, it is true the interval of Kendall's tau. We can conclude that Cotangent copula has much flexibility than the Cotangent hyperbolic copula.

Table 1. Interval of Kendall's Tau $(\tau)$, Lower $\left(\lambda_{L}\right)$ and Upper $\left(\lambda_{U}\right)$ tail dependences of trigonometric copulas

\begin{tabular}{ccccc} 
Copula & $\theta \in$ & $\tau \in$ & $\lambda_{U} \in$ & $\lambda_{L} \in$ \\
\hline Cot & $(0, \infty)$ & $(-0.2312,1)$ & $\{0\}$ & $(0,1)$ \\
Coth & $(0, \infty)$ & $(1 / 3,1)$ & $\{0\}$ & $\{1 / 2\}$ \\
\hline
\end{tabular}

\section{PARAMETER ESTIMATION OF COTANGENT COPULA: A SIMULATION STUDY}

In this section, we investigate the estimation of the parameter of Cotangent copula based on various methods. These are maximum Pseudo-likelihood, inverted Kendall's tau and minimum distance estimators based on the empirical process and Kendall process. Also, the Monte Carlo study is performed to measure the performance of four estimation methods.

Let $\left(X_{1}, Y_{1}\right), \ldots,\left(X_{n}, Y_{n}\right)$ be a bivariate random sample from copula $C(u, v)$. Suppose that Copula $C(u, v)$ has a density $c(u, v)$. defined by

$c(u, v)=\frac{\partial^{2} C(u, v)}{\partial u \partial v}, \quad u, v \in[0,1]$

then the pseudo-log-likelihood function is given by

$l(\theta)=\sum_{i=1}^{n} \log \left(c\left(F_{n}\left(x_{i}\right), G_{n}\left(y_{i}\right)\right)\right)$

where $F_{n}(x)$ and $G_{n}(y)$ are empirical distribution function defined, respectively, by

$F_{n}(x)=\frac{1}{n} \sum_{i=1}^{n} \boldsymbol{I}\left(X_{i} \leq x\right), \quad G_{n}(y)=\frac{1}{n} \sum_{i=1}^{n} \boldsymbol{I}\left(Y_{i} \leq y\right)$

where $\boldsymbol{I}$ denotes indicator function. The maximum pseudo-likelihood estimator is the value $\hat{\theta}_{n}^{M L E}$ that maximizes $l(\theta)$. Genest et al. [6] showed that $\hat{\theta}_{n}^{M L E}$ is a consistent estimator under some regularity 
conditions. They stated $\widehat{\theta}_{n}^{M L E}$ is consistent at independence. So, the consistency of $\widehat{\theta}_{n}^{M L E}$ may not be fulfilled as Kendall's tau increased. The function "optimize" in statistical programming language R is quite handy when maximizing likelihood function.

Another important parameter estimation method for Archimedean copula is inverted Kendall's tau estimator. Let $\tau=h(\theta)$ be Kendall's tau and let $\tau_{\mathrm{n}}$ be the empirical Kendall's tau based on a random sample of size $\mathrm{n}$. Then the moment estimator of $\theta$ denoted by $\hat{\theta}_{n}^{\tau}$ is given by $\hat{\theta}_{n}^{\tau}=h^{-1}\left(\tau_{n}\right) \cdot \sqrt{n}\left(\hat{\theta}_{n}^{\tau}-\theta\right)$ is asymptotically normal with zero mean. See, Kojadinovic and Yan [7].

Table 2. Estimated MSE scores of Cotangent copula parameter estimators with sample size $n=100,500$

\begin{tabular}{ccccccc}
\hline$n$ & $\tau$ & $\theta$ & $\hat{\theta}_{n}^{\tau}$ & $\hat{\theta}_{n}^{M L E}$ & $\hat{\theta}_{n}^{C v M_{C}}$ & $\hat{\theta}_{n}^{C v M_{K}}$ \\
\hline 0.1 & 0.52 & 0.0232 & 0.0403 & 0.0414 & $\mathbf{0 . 0 1 2 5}$ \\
& 0.2 & 0.76 & $\mathbf{0 . 0 3 4 7}$ & 0.0586 & 0.0657 & 0.0364 \\
& 0.3 & 1.05 & $\mathbf{0 . 0 4 5 7}$ & 0.0692 & 0.0879 & 0.0532 \\
& 0.4 & 1.43 & $\mathbf{0 . 0 7 9 0}$ & 0.1049 & 0.1668 & 0.0853 \\
& 0.5 & 1.95 & $\mathbf{0 . 1 1 7 9}$ & 0.1428 & 0.3015 & 0.1248 \\
& 0.6 & 2.71 & $\mathbf{0 . 2 0 0 9}$ & 0.2020 & 0.5733 & 0.2330 \\
& 0.7 & 3.95 & 0.4301 & $\mathbf{0 . 3 7 8 7}$ & 1.8115 & 0.4921 \\
& 0.8 & 6.37 & 1.1142 & $\mathbf{0 . 8 5 3 3}$ & 9.6958 & 1.4691 \\
\hline 0.1 & 0.52 & $\mathbf{0 . 0 0 4 0}$ & 0.0124 & 0.0049 & 0.0041 \\
& 0.2 & 0.76 & $\mathbf{0 . 0 0 6 1}$ & 0.0164 & 0.0075 & 0.0067 \\
& 0.3 & 1.05 & $\mathbf{0 . 0 0 9 8}$ & 0.0215 & 0.0120 & 0.0110 \\
& 0.4 & 1.43 & $\mathbf{0 . 0 1 3 9}$ & 0.0262 & 0.0176 & 0.0153 \\
& 0.5 & 1.95 & $\mathbf{0 . 0 2 1 4}$ & 0.0344 & 0.0287 & 0.0242 \\
& 0.6 & 2.71 & $\mathbf{0 . 0 3 5 6}$ & 0.0461 & 0.0522 & 0.0413 \\
& 0.7 & 3.95 & $\mathbf{0 . 0 6 6 6}$ & 0.0690 & 0.1117 & 0.0793 \\
& 0.8 & 6.37 & 0.1537 & $\mathbf{0 . 1 3 5 4}$ & 0.3410 & 0.1953 \\
\hline
\end{tabular}

Weiß [8] investigated the different minimum-distance estimators for copula parameters. The first one is minimum-distance estimators based on the empirical copula process. This method is based on the empirical process

$\mathbb{C}_{n}=\sqrt{n}\left(C_{n}-C_{\theta}\right)$

where $C_{n}$ is empirical copula defined by

$C_{n}(u, v)=\frac{1}{n} \sum_{i=1}^{n} \boldsymbol{I}\left(U_{i, 1}<u, V_{i, 1}<v\right)$,

and $C_{\theta}$ is the hypothesised copula. Cramer-von-Mises statistic based on $\mathbb{C}_{n}$ is defined by

$C v M_{C}=\int_{0}^{1} \int_{0}^{1} \mathbb{C}_{n}^{2} d C_{n}$

The minimum distance estimators based on the empirical copula process is given by 


$$
\hat{\theta}_{n}^{C v M_{C}}=\underset{\theta \in \Theta}{\operatorname{argmin}} C v M_{C}
$$

The convergence of the empirical copula process is investigated in Genest et al. [9]

The second type of Minimum distance estimator is based on Kendall process. Genest and Rivest [10] investigated empirical estimate of Kendall distribution function $(t)=t-\frac{\varphi(t)}{\varphi^{\prime}(t)}$. For the estimation of the random variable $\mathrm{T}=\mathrm{H}(\mathrm{x}, \mathrm{y})$, univariate distribution function $K(T)=P(H(x, y) \leq t)=P(C(u, v) \leq t)$ should be estimated on the interval $[0,1]$. Then a nonparametric estimation of $K_{n}(t)$ is given by

$K_{n}(t)=\frac{1}{n} \sum_{i=1}^{n} I\left(T_{i} \leq t\right)$

Also, we note that Susam and Ucer [11] proposed estimation of $\mathrm{K}(\mathrm{t})$, which is a continuous approximation of $\mathrm{K}_{\mathrm{n}}(\mathrm{t})$. Genest et al. [12] defined Kendall process

$\mathbb{K}_{n}=\sqrt{n}\left(K_{n}-K\right)$

Cramer-von-Mises statistic based on $\mathbb{K}_{\mathrm{n}}$ is defined by

$$
C v M_{K}=\int_{0}^{1} \mathbb{K}_{n}(t)^{2} d K_{n}(t)
$$

The minimum distance estimator based on the Kendall process is given by

$$
\hat{\theta}_{n}^{C v M_{K}}=\underset{\theta \in \Theta}{\operatorname{argmin}} C v M_{K}
$$

The convergence of the empirical process $\mathbb{K}_{n}$ is investigated in Genest and Rivest [10].

Monte Carlo simulation study was conducted to compare Inversion of Kendall's tau estimator, Maximum pseudo-likelihood estimator, and Minimum distance estimators of dependence parameter $\theta$ of Cotangent copula. The primary purpose is achieved by comparing the true parameter with the parameters estimated with the four estimation methods. The data are generated from Cotangent copula with Kendall's Tau 0.2, $0.3, \ldots, 0.8$ and sample of sizes $n=100,500$. Table 2 presents the estimated Mean squared error (MSE) scores of the four estimation methods. We can conclude that Inverted Kendall's tau method outperforms the other estimation methods for sample size $n=100$ and $\tau<0.7$. For $\tau \geq 0.7$ and $n=100$, the maximum Pseudolikelihood estimator is the best one. Inverted Kendall's tau method has lower MSE scores for sample size $n=500$ and $\tau<0.7$. Also, for $\tau \geq 0.7$ and $n=500$, the maximum Pseudo-likelihood estimator outperforms the others.

\section{REAL DATA EXAMPLE}

In this section, we fit Cotangent copula and the Cotangent hyperbolic copula to two data sets used in Najjari et al. [3]. According to this paper, two data sets share $n=113$ annual (May-October) maxima of the Vltava river (Bohemia) flow rate (measured above the dam Kamyk in period 1890-2007) for which the corresponding flood volume was computed (from daily flow averages) by two methods.

Scatter plots of the two data set are shown in Figure 4. It demonstrates obvious dependence structures (especially lower tail dependence) between involved random variables. Copulas were fitted to data sets using the pseudo-likelihood method as described in Section 3. Also, Goodness of fit was tested by $C v M_{K}$ defined in Section 3. The goodness of fit results of the two data sets are shown in Table 3. Also, empirical and fitted $\lambda$ functions are visualized in Figure 5. We select $\lambda$ function as a comparison of the goodness of fit because $\lambda$ function has better visualization than the generator function. See, Michiels et al. [5]. 


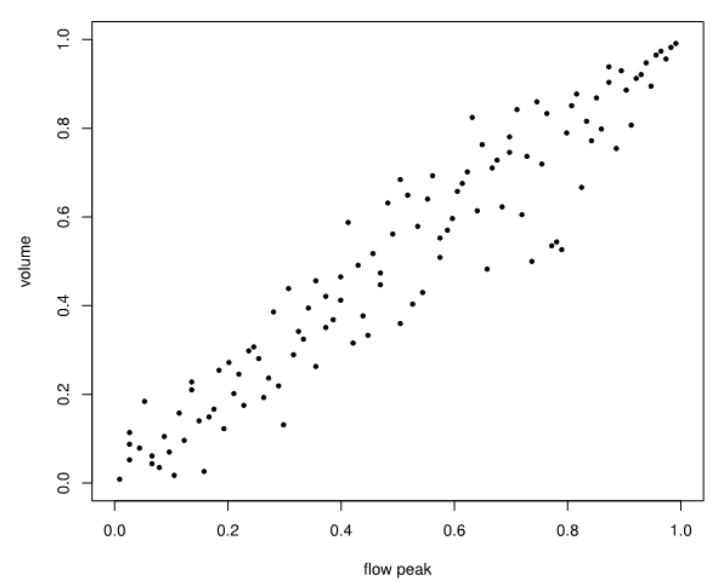

(a) fixed-width

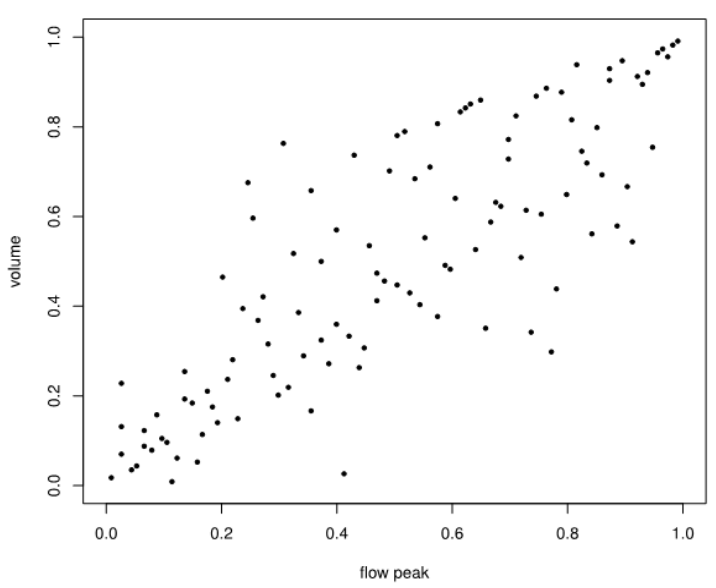

(b) variable-width flood wave

Figure 4. Scatterplots of the data sets

From Table 3, two types of copula provide a strong fit. In addition to, Cotangent copula outperforms the Cotangent hyperbolic copula for the data set variable-width flood wave. When Figure 5(b) examined, Cotangent copula has a better fit at left tails of the data set than Cotangent hyperbolic copula. From Figure (5a), it is also true for the data sets fixed-width. On the other hand, Cotangent hyperbolic copula has a better fit at the right tail. The goodness of fit results for the most used one-parameter families is derived in Bal and Najjari [2].

Table 3. The goodness of fit results for two data set

\begin{tabular}{lllll}
\hline Data & Copula & $\theta$ & CvM & P-value \\
\hline fixed-width & Cot & 6.9891 & 0.0422 & 0.1384 \\
& Coth & 9.0425 & 0.0346 & 0.2358 \\
\hline variable-width flood wave & Cot & 3.2354 & 0.0415 & 0.5111 \\
& Coth & 4.1854 & 0.0591 & 0.1785 \\
\hline
\end{tabular}

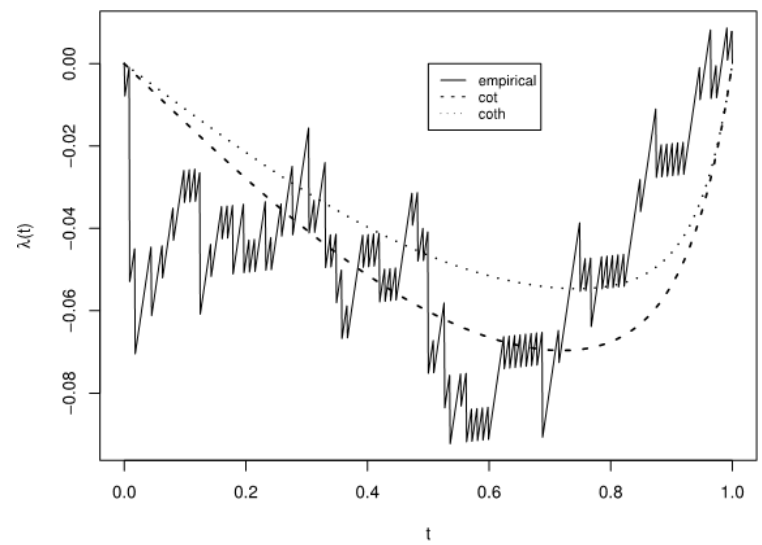

(a) fixed-width

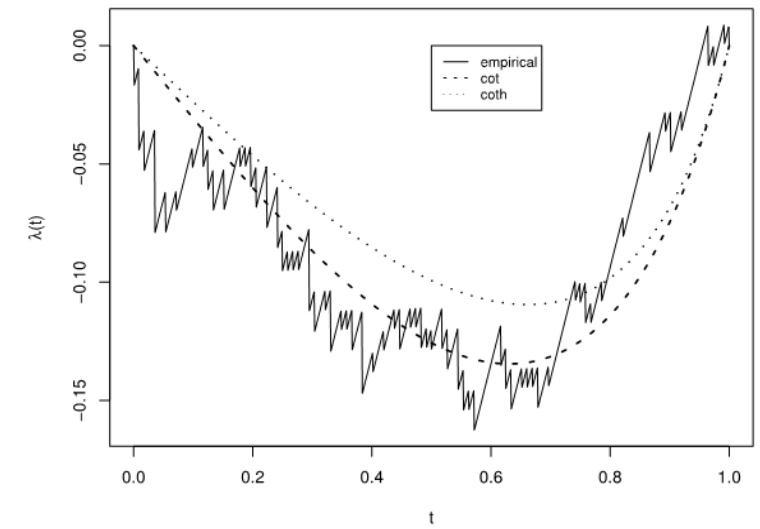

(b) variable-width flood wave

Figure 5. Visualization of the goodness-of-fit of the Cotangent and Cotangent hyperbolic copula based on $\lambda$ function

\section{REAL DATA EXAMPLE}

Cotangent functions have been proposed as a generator function of the new family. Dependence characteristics such as Kendall's tau, Upper tail dependence, and Lower tail dependence of this new family are derived. Also, the estimation performance of the dependence parameter $\theta$ investigated via Monte Carlo 
simulation study for four estimation methods. According to the Monte Carlo simulation study, Inverted Kendall's tau method has better performance than the others in almost all cases. We modeled the dependence structure of the two data sets using two trigonometric copulas. Cotangent copula performs well in Real application data. Also, we note that more parameterized generators allow for better flexibility while modeling the copula. One can investigate the generator function $\varphi(t)=\cot (\beta t)^{\theta}-\cot (\beta)^{\theta}$ and related dependence properties with valid parameters $\theta$ and $\beta$ for $t \epsilon[0,1]$.

\section{CONFLICTS OF INTEREST}

No conflict of interest was declared by the author.

\section{REFERENCES}

[1] Sklar, A., "Fonctions de Répartition à n Dimensions et Leurs Marges", Publications de 1'Institut Statistique de l'Université de Paris, 8:229-231, (1959).

[2] Bal, H. and Najjari, V., "Archimedean copulas family via hyperbolic generator", Gazi University Journal of Science, 26:195-200, (2013).

[3] Najjari, V., Bacigal, T. and Bal, H., "An archimedean copula family with hyperbolic cotangent generator", International Journal of Uncertainty Fuzziness and Knowledge-Basedd Systems, 20 (5): 761-768, (2014).

[4] Bagdasaryan, A., "A Note on the ${ }_{1}^{2} F$ Hypergeometric Function”, Journal of Mathematics Research, 2(3): 71-77, (2010).

[5] Michiels F., Koch, I. and De Schepper A., "How to improve the fit of Archimedean copulas by means of transforms", Statistical Papers, 53:345-355, (2012).

[6] Genest C., Molina J. and Lallena, J., "De l'impossibilité de construire des lois à marges multidimensionnelles données à partir de copules", Comptes rendus de l'Acadmie des Sciences, 320: 723-726, (1995).

[7] Kojadinovic, I. and Yan, J., "Comparison of three semiparametric methods for estimating dependence parameters in copula models", Insurance: Mathematics and Economics, 47:52-63, (2010).

[8] Weiß, G., "Copula parameter estimation by maximum-likelihood and minimum-distance estimators: a simulation study", Computitional Statistics, 26:31-54, (2011).

[9] Genest, C., Remillard, B. and Beaudoin, D., "Goodness-of-fit tests for copulas: A review and a power study”, Insur Math Econ, 44:199-213, (2008).

[10] Genest, C. and Rivest, L., "Statistical inference procedures for bivariate Archimedean copulas", Journal of the American Statistical Association, 88: 1043-1043, (1993).

[11] Susam, S.O. and Ucer, B.H., "Testing independence for Archimedean copula based on Bernstein estimate of Kendall distribution function", Journal of Statistical Computation and Simulation, 88(13): 2589-2599, (2018).

[12] Genest, C., Quessy, J.F. and Remillard, B., "Goodness-of-fit procedures for copula models based on the probability integral transform”, Scandinavian Journal of Statistics, 33:337-366, (2006). 